\title{
Spectator charge splitting of directed flow in heavy ion collisions
}

\author{
Antoni Szczurek ${ }^{* a, b}$ and Andrzej Rybicki ${ }^{a \dagger}$ \\ a Institute of Nuclear Physics, PL-31-342 Kraków \\ ${ }^{b}$ University of Rzeszów, PL-35-959 Rzeszów \\ E-mail: antoni.szczurek@ifj.edu.pl, andrzej.rybicki@ifj.edu.pl
}

We estimate the effect of the spectator charge on distortion of single charged pion distributions as well as on azimuthal anisotropies in heavy ion collisions. A large electromagnetic effect on directed flow $v_{1}$ is predicted in good agreement with existing WA98 as well as RHIC data. This effect results in a splitting of $v_{1}$ for positive and negative pions. Detailed analysis of this phenomenon may provide new information on the collision dynamics.

The European Physical Society Conference on High Energy Physics -EPS-HEP2013 18-24 July 2013

Stockholm, Sweden

* Speaker.

${ }^{\dagger}$ AS thanks organizers of EPS2013 conference for very good organization and atmosphere. 


\section{Introduction}

Noncentral nucleus-nucleus collisions unambigously lead to azimuthal asymmetries and presence of spectators. Azimuthal correlations between particles and the reaction plane are one of the main subjects of study in heavy ion collisions. They provide information about collective effects. On the other hand, the presence of charged fast moving spectators generates strong electromagnetic fields. The resulting electromagnetic effects modify single particle spectra [1]. Recently, we discussed how these electromagnetic effects influence also azimuthal correlations [2].

Our first constatation [1] that the EM fields generated by the remnants (spectators) of peripheral collisions distort the charged pion spectra was supported by precise NA49 experimental data at $\sqrt{s_{N N}}=17.3 \mathrm{GeV}$ [3]. Spectacular effects were predicted and observed:

- A dip in $\pi^{+}$density at pion $x_{F}=0.15$;

- An accumulation of strength for $\pi^{-}$at $x_{F}=0.15$.

In this presentation we first remind the effect of distortions of single particle spectra, and then discuss the influence of electromagnetic fields generated by the spectators on directed flow. Results of theoretical calculations are compared to experimental data $[4,5]$. The results included in this presentation are partially based on our precedent works [1,2].

\section{Sketch of the model}

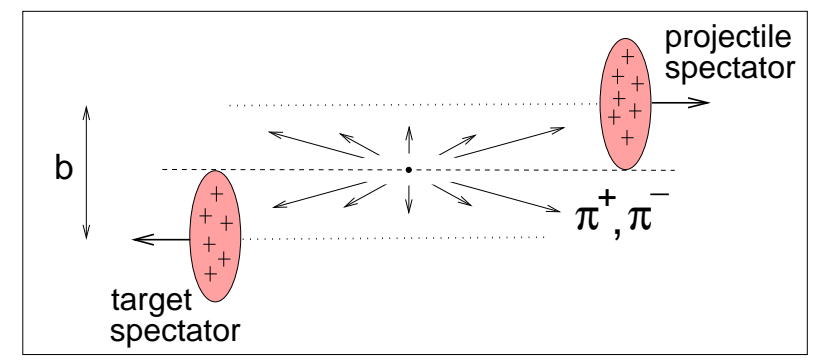

Figure 1: Our simplified view of a Pb+Pb collision. The hypothetical pion emission region is reduced to a single point in position space.

In Fig.1 we present a general view of our model of the peripheral collision. The situation can be summarized as follows:

- the collision takes place at a given impact parameter $b$.

- the two charged spectator systems follow their initial path.

- the participating system evolves until pions are produced.

- charged pion trajectories are modified by the EM interaction.

- the spectator systems undergo a complicated, not fully understood, nuclear deexcitation/fragmentation process. 
The initially produced charged pions are subjected to the EM field of the two spectator systems. The spectator velocity remains constant and identical to the velocity of the parent $\mathrm{Pb}$ ion. $\mathrm{We}$ choose the overall $\mathrm{CM}$ system to calculate the evolution of pion trajectories. We note that for symmetric $\mathrm{Pb}+\mathrm{Pb}$ collisions, this is also the $\mathrm{N}+\mathrm{N}$ CM system.

The azimuthal correlations are usually quantified in terms of the Fourier coefficients of the azimuthal distribution of the outgoing particles with respect to the reaction plane:

$$
v_{n} \equiv<\cos \left[n\left(\phi-\Psi_{r}\right)\right]>,
$$

where $\phi$ is the azimuthal angle of the emitted particle (pion), while $\Psi_{r}$ is the orientation of the reaction plane defined by the impact parameter vector $\vec{b}$.

The first order coefficient

$$
v_{1} \equiv<\cos \left(\phi-\Psi_{r}\right)>
$$

reflects the sideward collective motion and is known as directed flow. There exist rich data on $v_{1}$ from FOPI, E877, WA98, NA49, STAR, ALICE and other experiments. With some exceptions which will be discussed in this paper, these data are obtained for both charges of pions.

What is known about directed flow? The underlying symmetry imposes its dependence on particle rapidity as an asymmetric function $\left(v_{1}(y)=-v_{1}(-y)\right)$. The Glauber approach gives tilted initial conditions which leads to tilted pressure and hydrodynamics produces final $v_{1}$ [6]. The effect drops with collision energy.

The effects of the EM interaction discussed in this paper $\left(v_{1}^{\pi, E M}\right)$ will add up to these imposed by the strong force $\left(v_{1}^{\text {flow }}\right)$. For the final state values of $v_{1}$ observed in experiment, we expect as a first approximation:

$$
\begin{aligned}
& v_{1}^{\pi^{+}} \approx v_{1}^{\text {flow }}+v_{1}^{\pi^{+}, E M}, \\
& v_{1}^{\pi^{-}} \approx v_{1}^{\text {flow }}+v_{1}^{\pi^{-}, E M} .
\end{aligned}
$$

For simplicity, in this presentation we shall discuss only the pure electromagnetic component of directed flow. We note that this simplification does not affect the generality of the conclusions presented in this paper.

\section{Distortions of inclusive spectra of charged pions}

First we wish to remind how the electromagnetic effects distort inclusive spectra of pions. In Fig. 2 we show the ratio of cross sections for $\pi^{+}$and $\pi^{-}$production as a function of pion $x_{F}$ for different pion emission times defined in Ref.[1].

In Fig.3 we show similar ratio as a function of $x_{F}$ for fixed values of transverse momenta.

\section{Splitting of directed flow of charge pions}

In Fig.4 we show the comparison of the EM-induced directed flow $v_{1}$ obtained seperately for each of the two spectators, compared to that obtained when both spectators are included in the simulation. The figure demonstrates the additivity of these two components in the total EM effect.

In Fig. 5 we show the EM effect on $v_{1}$ as a function of rescaled rapidity for two different values of the pion emission time. 


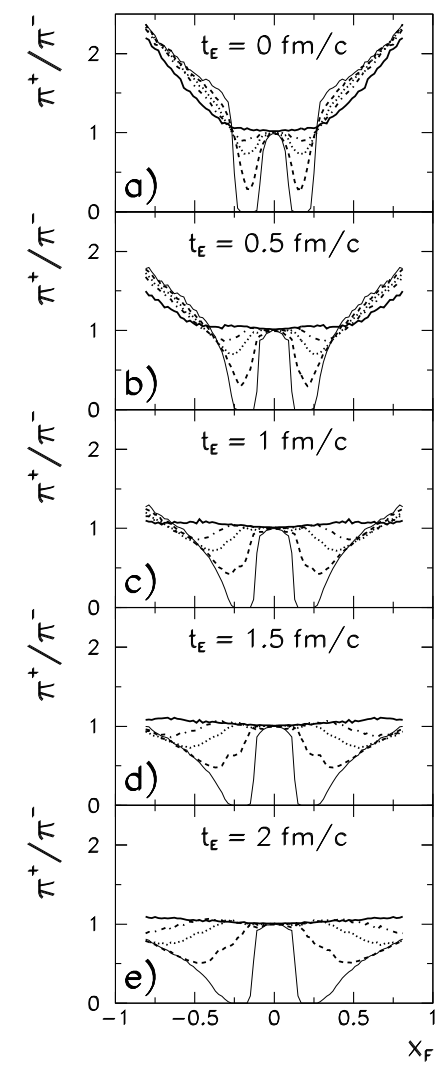

Figure 2: Ratio of density of produced $\pi^{+}$over produced $\pi^{-}$in the final state of the peripheral $\mathrm{Pb}+\mathrm{Pb}$ reaction, obtained for five values of the pion emission time $t_{E}$. The $\pi^{+} / \pi^{-}$ratio is drawn as a function of $x_{F}$ at $p_{T}=25 \mathrm{MeV} / \mathrm{c}$ (thin solid), $75 \mathrm{MeV} / \mathrm{c}$ (dash), $125 \mathrm{MeV} / \mathrm{c}$ (dot), $175 \mathrm{MeV} / \mathrm{c}$ (dash-dot), and $325 \mathrm{MeV} / \mathrm{c}$ (thick solid).

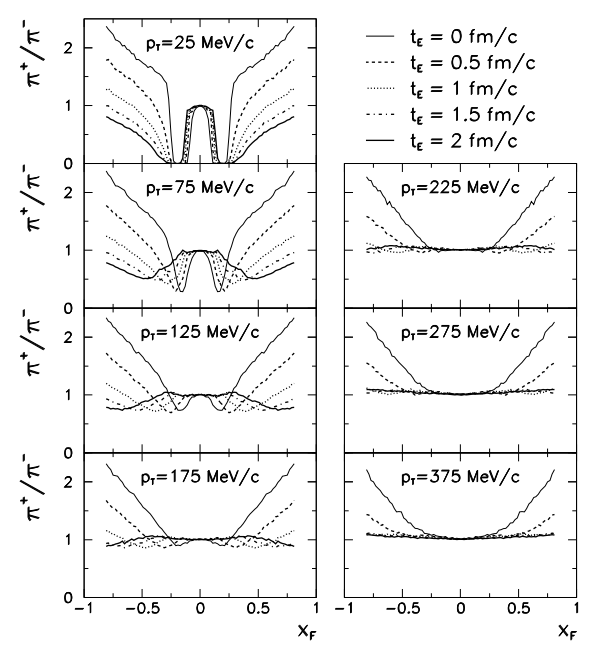

Figure 3: The $\pi^{+} / \pi^{-}$cross section ratio for the peripheral $\mathrm{Pb}+\mathrm{Pb}$ reaction, shown at fixed values of $p_{T}$ as a function of $x_{F}$. The five considered values of the pion emission time $t_{E}$ are differentiated by means of different line types. 

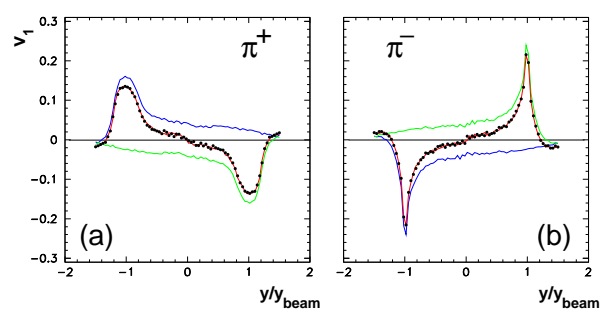

Figure 4: (Color online) Spectator-induced electromagnetic effect on directed flow of (a) $\pi^{+}$and (b) $\pi^{-}$, in peripheral $\mathrm{Pb}+\mathrm{Pb}$ collisions at $\sqrt{s_{N N}}=17.3 \mathrm{GeV}$. The green solid curve shows the directed flow induced electromagnetically by the right $(\mathrm{R})$ spectator. The blue solid curve shows the directed flow induced electromagnetically by the left (L) spectator. Black dots show the result of the addition of these two curves. The red solid curve displays the result of the simulation including both spectators. Note: all the simulations assume the pion emission time $t_{E}=0 \mathrm{fm} / \mathrm{c}$.

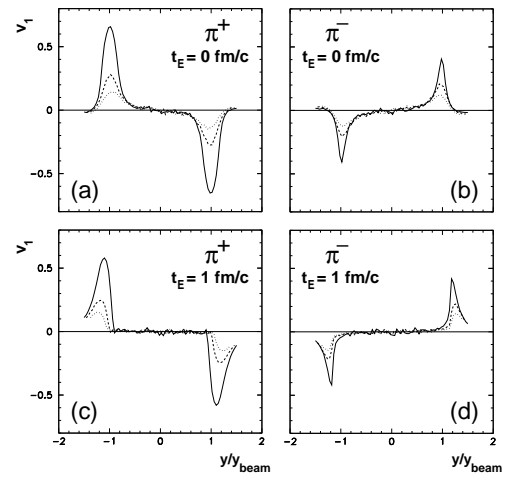

Figure 5: Spectator-induced electromagnetic effect on directed flow of $\pi^{+}$and $\pi^{-}$in peripheral $\mathrm{Pb}+\mathrm{Pb}$ collisions at $\sqrt{s_{N N}}=17.3 \mathrm{GeV}$, shown at fixed values of pion transverse momentum: $p_{T}=75 \mathrm{MeV} / \mathrm{c}$ (solid), $p_{T}=125 \mathrm{MeV} / \mathrm{c}$ (dashed), $p_{T}=175 \mathrm{MeV} / \mathrm{c}$ (dotted). The top and bottom panels correspond to different values of the pion emission time $t_{E}$.

Fig. 6 shows the experimental data of the WA98 collaboration, superimposed with the rapiditydependence of the electromagnetically-induced directed flow as obtained from our work. For the latter, three values of the pion emission time are assumed: $t_{E}=0,0.5$, and $1 \mathrm{fm} / \mathrm{c}$. Our results are integrated over $p_{T}$ from 0 to $1 \mathrm{GeV} / \mathrm{c}$. The curves corresponding to $t_{E}=0$ are the same as in Fig. 4 .

In Fig. 7 we show how $v_{1}$ depends on the emission time. The shorter emission time the larger $v_{1}$. This figure, when compared to experimental data, can allow to extract the emission time.

In Fig. 8 we show the best fit of the emission time to STAR data [5]. We note that the average directed flow $\frac{v_{1}^{\pi^{+}}+v_{1}^{\pi^{-}}}{2}$ is subtracted from the STAR data in order to isolate its electromagnetic component as discussed above. From this fit we conclude that pions are produced at an (average) distance of about $3 \mathrm{fm}$ from the spectator system. A more detailed discussion will be given in [7].

\section{Conclusions and Outlook}

Our investigation concerning electromagnetic effects caused by fast moving spectators can be summarized as follows: 


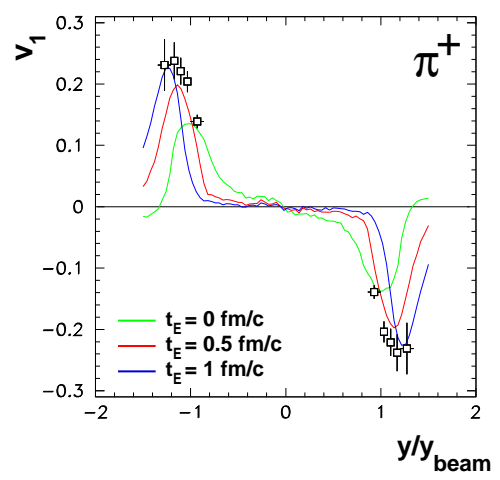

Figure 6: Comparison between experimental data on directed flow of positive pions obtained by the WA98 experiment [4]), and our simulation of electromagnetically-induced directed flow of $\pi^{+}$. The three curves correspond to three different values of the assumed pion emission time $t_{E}$.
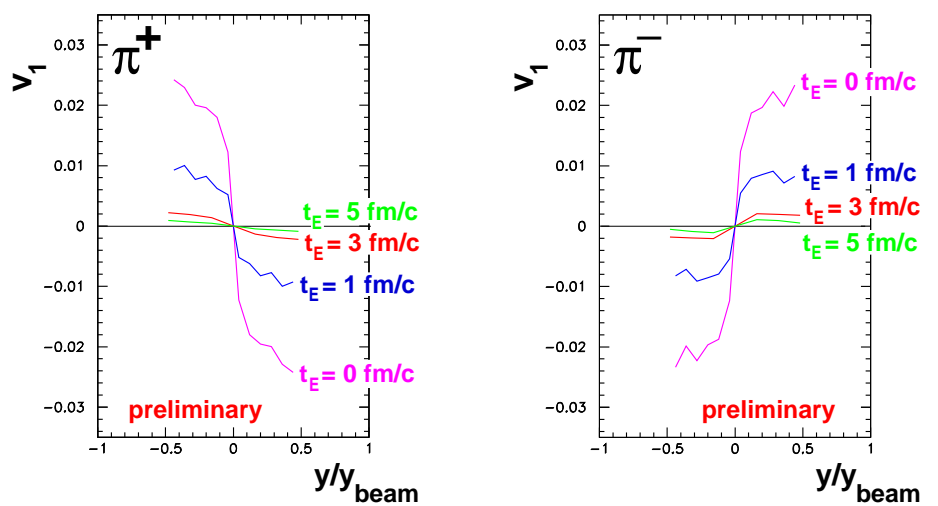

Figure 7: Emission time dependence of directed flow. The simulation has been made for $A u+A u$ collisions at $\sqrt{s_{N N}}=7.7 \mathrm{GeV}$.
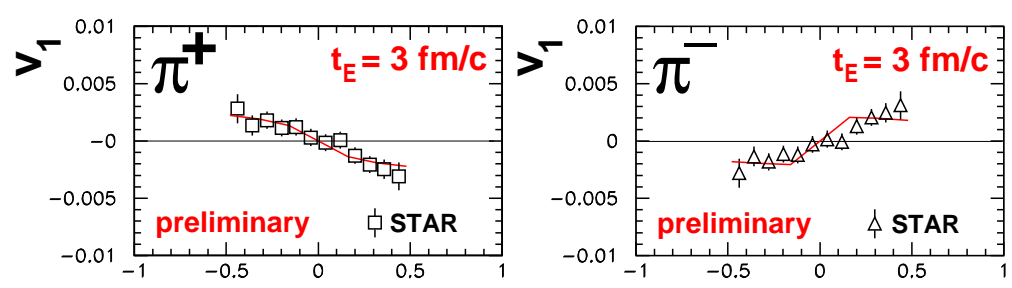

Figure 8: Fitting the emission time to STAR data [5].

- The EM interaction caused by the moving remnant charge produces visible distorsions in the final state distributions of $\pi^{+}$and $\pi^{-}$.

- The main feature of this electromagnetic effect is a big dip in the $\pi^{+}$density distribution at low transverse momenta in the vicinity of $x_{F} \approx \pm 0.15$, accompanied by a substantial increase of $\pi^{-}$density in the same region.

- The effect is clearly sensitive to initial conditions and carries interesting information on the 
mechanism of the non-perturbative particle production process, and in particular on its evolution in space and time.

- Our study demonstrates the importance of new, double-differential data on the $x_{F}$ and $p_{T^{-}}$ dependence of pion production in peripheral nucleus+nucleus collisions.

- Electromagnetic fields generated by charged, fast spectators lead to extra azimuthal distortions and contribute to directed flow.

- The above effect is opposite for $\pi^{+}$and $\pi^{-}$and leads to a splitting of $v_{1}$. This splitting is superimposed on other effects (like hydrodynamics).

- This effect is confirmed by the WA98 and STAR data.

- The splitting strongly depends on the emission time of pions and can be therefore used to measure the emission time.

- The splitting depends on the transverse momentum of pions.

The topic discussed here requires further investigations. In particular:

- Precise data for $\pi^{+}$and $\pi^{-}$and different energies are needed.

- The dependence on rapidity and transverse momentum should be analyzed.

- Realistic modelling of the source is badly needed.

- A procedure to extract emission time would be very useful and provide a complementary information to, e.g., HBT measurements.

- The evolution time of spectator systems should be better understood.

- Other harmonics are also subjected to spectator EM splitting.

- Exploration of the above electromagnetic effects as a function of particle species, rapidity, transverse momentum and centrality will provide valuable insight into the non-perturbative dynamics of the heavy ion collision.

\section{Acknowledgments}

This work was supported by the Polish National Science Centre (on the basis of decision no. DEC-2011/03/B/ST2/02634).

\section{References}

[1] A. Rybicki and A. Szczurek, Phys. Rev. C75 (2007) 054903.

[2] A. Rybicki and A. Szczurek, Phys. Rev. C87 (2013) 054909.

[3] A. Rybicki, Acta Phys. Polon.B42 (2011) 867.

[4] H. Schlagheck (WA98 Collaboration), Nucl. Phys. A 663, 725 (2000).

[5] Y. Pandit (STAR Collaboration), Acta Phys. Polon. Supp. 5, 439 (2012).

[6] P. Bożek and I. Wyskiel, Phys. Rev. C81 (2010) 054902; P. Bożek, Phys. Rev. C85 (2012) 034901.

[7] A. Rybicki and A. Szczurek, work in preparation. 